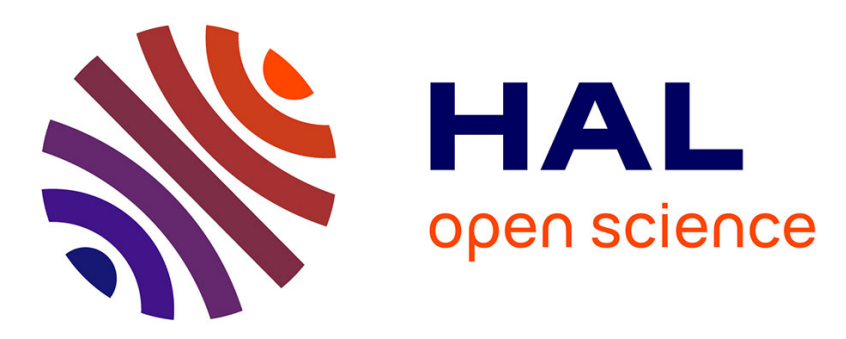

\title{
Stiffness rendering on soft tangible devices controlled through inverse FEM simulation
}

Frédérick Largillière, Eulalie Coevoet, Mario Sanz Lopez, Laurent Grisoni, Christian Duriez

\section{- To cite this version:}

Frédérick Largillière, Eulalie Coevoet, Mario Sanz Lopez, Laurent Grisoni, Christian Duriez. Stiffness rendering on soft tangible devices controlled through inverse FEM simulation. International Conference on Intelligent Robots and Systems - IROS 2016, Oct 2016, Daejeon, South Korea. hal-01386787

\section{HAL Id: hal-01386787 \\ https://hal.inria.fr/hal-01386787}

Submitted on 24 Oct 2016

HAL is a multi-disciplinary open access archive for the deposit and dissemination of scientific research documents, whether they are published or not. The documents may come from teaching and research institutions in France or abroad, or from public or private research centers.
L'archive ouverte pluridisciplinaire HAL, est destinée au dépôt et à la diffusion de documents scientifiques de niveau recherche, publiés ou non, émanant des établissements d'enseignement et de recherche français ou étrangers, des laboratoires publics ou privés. 


\title{
Stiffness rendering on soft tangible devices controlled through inverse FEM simulation
}

\author{
Frédérick Largillière* Eulalie Coevoet Mario Sanz-Lopez Laurent Grisoni Christian Duriez*
}

\begin{abstract}
Haptic rendering of soft bodies is essential in medical simulations of procedures such as surgery or palpation. The most commonly used approach is to recreate the sense of touch using a specific design and control of a robotic arm. In this paper, we propose a new approach, based on softrobotics technology. We create a tangible deformable device that allows users to "touch" soft tissues and perceive mechanical material properties, in a realistic manner. The device is able to dynamically provide user touch with different stiffness perceptions, thanks to actuators placed at the boundaries. We introduce a control algorithm, based on inverse Finite Element Analysis, which controls the actuators in order to recreate a desired stiffness that corresponds to the contact with soft tissues in the virtual environment. The approach uses antagonistic actuation principle to create a wide range of stiffness. We validate our algorithm and demonstrate the method using prototypes based on simple mechanisms.
\end{abstract}

\section{INTRODUCTION}

Aside visual and acoustic senses, touch is a human sense that we extensively use in our relation to the world. In the context of virtual reality, the use of haptic rendering improves the immersion by adding the sense of touch [1]. Being able to touch, in a realistic manner, the result of a virtual reality-based simulation, in a way that would be as rich as in real world, is still a pure fantasy. Actually several physical properties of real world objects could be rendered such as contact force, friction, texture [2] among others. Haptic rendering (Kinesthetic rendering) is usually performed using a reversible robotic arm or an exoskeleton on which the torque of the motors is controlled in order to reproduce the desired force, computed in a simulation of the virtual environment. Obtaining a stable and transparent haptic rendering of complex virtual environments has been a challenge for nearly two decades. The reason is that the control of such devices requires high update rates that impose high constraints on computation time for the simulation.

For medical applications, haptic rendering of soft tissues has been extensively studied, particularly due to the difficulty of modeling soft tissue deformations [3]. But haptic rendering techniques dedicated to soft tissues and more generally to deformable objects have progressively emerged [4] [5] [6].

In this work, we build on these development but we explore a drastically different approach, since we propose to change the way the interaction forces with deformable bodies are transmitted to the user: Instead of using rigid robotic arms, the approach is to use tangible devices made

\footnotetext{
* indicates the corresponding authors, email: firstname.name@inria.fr The authors are with INRIA and University of Lille, Computer Science and Control Laboratory (CRISTAL) joint with CNRS (UMR 9189) and Centrale Lille.
}

of soft material. This type of tangible technique has emerged recently [7], but the design and control of such devices remains an open problem.

In our work, the interfaces are based on a principle of antagonistic actuation to allow a control of their apparent stiffness. More precisely, this project aims at rendering the contact with soft tissues through a new approach: where this problem is usually addressed though indirect haptics (i.e tools that are virtually in contact with the tissues and haptic rendering through PHANTOM(C) Omni-like interfaces), we want here to reproduce a direct touch on the tissue through a soft apparatus on which we can regulate the apparent stiffness.

In order to control this apparent stiffness, we make use of recent results in the control through inverse FEM simulation of soft robots [8]. This control algorithm allows to compute forces to be applied on a small set of points of soft structures so that specific points move to a desired position. In this paper, we extend the approach to force and, thus, stiffness control. The simulation is coded in SOFA[9], an open-source framework that contains fast implementations of FEM as well as optimization methods for collision response and mechanical interactions with haptic feedback. SOFA is both used to simulate a virtual environment containing a soft body that we want to render with haptics and also to control, using an inverse model, the apparent stiffness of the device. This work has been applied on two prototypes made of silicone: the first one is stiffened through antagonistic tension forces and the second one uses pneumatic and tendons actuations in an antagonistic manner.

In brief, the paper presents a generic algorithm to control the apparent stiffness of soft devices with antagonistic actuation. We show that this algorithm can be used to create haptic rendering of soft bodies in an interesting way. In section 2 , related work on haptic rendering on tangible device is quickly introduced. An overview of the concepts used in our methods is presented in section 3 while the methodology to obtain a numerical formulation of the problem is developed in section 4. Section 5 presents the control algorithm used and a practical use of this methodology on 2 prototypes based on simple mechanisms forms the section 6. Finally, a discussion on the developed method makes the section 7 and a conclusion and future perspectives are discussed in section 8 .

\section{TOWARDS SOFT TANGIBLE RENDERING}

In this section, we briefly present some related work to haptic with tangible rendering and soft robotics. First, the 
use of tangible approach did not only come from a problem of haptic rendering. Tangible user interfaces are presented in [10]. In this approach, the aim is that the world will be an interface so the user is immersed with interfaces. Within the physical space around the user, tangible devices allow the user to center his attention with graspable media. The physical properties of the media do not change.

In [11] it is proposed to dynamically change the user interface thanks to a shape display called inForm. The user can program different types of shape or behavior that are displayed on a matrix of 900 pins actuated in height. The device can provide an independent haptic feedback on each of these rigid pins, but the system can hardly simulate a deformable environment. In [12], metal liquid is used to change the volume and the weight of a device so that the properties of the media can change during the user experience.

On the other hand, soft robotics is an emerging field which opens a new way of designing robots and actuated systems with safe and direct contact with humans. Soft robotics represent a vast set of new opportunities for tangible rendering thanks to its inner properties of compliance which prevents harmful collision response. The use of soft-robotic for haptic display has been investigated in [13], and [14] proposes a new kind of actuators that allow to create tangible devices from a vast range of objects while making them benefit from the compliance of soft actuation. Stiffness control is also a topic where soft robotics present advantages through their compliance to antagonistic forces [15] or the embedding of Shape Memory Alloys [16]

In this work, we target the development of anatomical soft robots: soft-robot with the shape of organs, equipped with sensors to measure the contact forces, and actuators to be able to stiffen the walls and recreate natural motion of softtissues. The main advantage of this approach would be the possibility to use standard surgical/medical devices during training and obtain haptic rendering through contact with environment, like in real life.

\section{OVERVIEW OF THE METHOD}

The haptic rendering developed in this paper is based on two concepts. First, it is necessary to control a contact point both in position and in stiffness. This leads to rendering a desired stiffness while maintaining the position where the user displaced the point where his finger is in contact with the interface. In order to obtain this result, we control the position of the point using antagonistic actuation forces. While the resultant of these forces is a null force when at the equilibrium of the desired position, we can adjust their intensity freely. When pushed out of the position equilibrium, a low intensity in force will only be a weak obstacle to overcome and will result in a low apparent stiffness, while a high intensity will be harder to counter, resulting in a high apparent stiffness.

In addition to this mechanical concept, it is also necessary to obtain numerically the values of actuation that will create the desired feeling when in contact with the interface. To
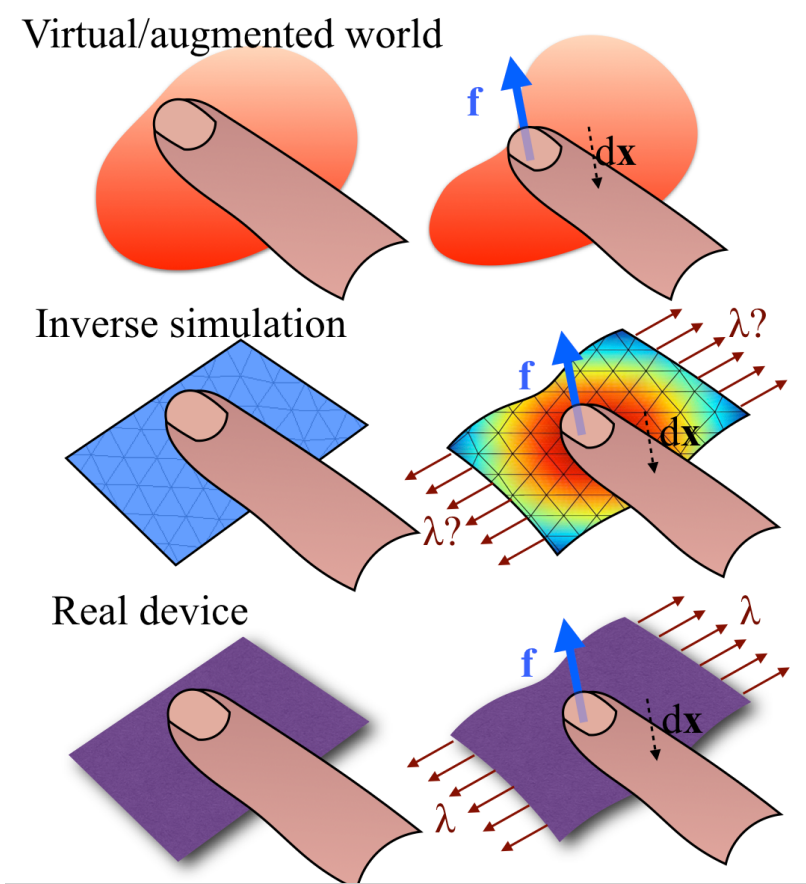

Fig. 1. Concept of stiffness rendering controlled through FEM inverse resolution. (Top) A virtual finger touches a simulated material with a penetration depth $\mathrm{dx}$ (measured by the device) which creates a reaction force $\mathrm{f}$ from contact with the virtual soft object. (Middle) An inverse problem is solved based on the Finite Element simulation of the real device to obtain the required actuation $\lambda$ to produce the reaction force f. (Bottom) The value of actuation is applied on the real device to produce the experienced force $\mathrm{f}$ from the pushing depth $\mathrm{dx}$ created by the user and measured on the device

achieve this result, we propose the use of a resolution of an inverse problem based on Finite Element simulation of the real interface. This methodology, firstly developed in [8] allows to find the displacement of actuators that will generate the desired motion or force at the effector (see fig.1). In this paper, we slightly modified the input of the algorithm in order to control the apparent stiffness of the soft interface with antagonistic actuation. We also show that the approach can be extended to hyperelastic materials, which is useful for improving the precision in the case of large deformations. In the following section, we provide some details about this inverse problem algorithm.

\section{INVERSE PROBLEM FOR STIFFNESS CONTROL}

The main idea developed in this project is to control the intensity of the contact force between the finger and the soft interface: we want to recreate artificially a profile of force that corresponds to the contact force with the deformable object in the simulation. As we want to adapt the profile of force when pushing on the device, it could also be considered as equivalent to a control of the apparent stiffness of the device.

In this context, we need to adapt and control the interaction force on a specific point (that we will call effector) of the soft device and take into account the coupling with the forces that are applied by the actuators. To achieve this goal, we will make use of the algorithm developed in [8] with 
the Quadratic Programming (QP) formulation provided in [17]. This algorithm is based on real-time simulation of the mechanical behavior of the soft structure using the Finite Element Method (FEM). The model provides the rigidity of the structure and the deformations created by actuators. An inverse resolution is performed to get the input forces that create the desired forces or displacements on the effectors. In the following paragraphs, we present the needed technical details of this algorithm before explaining how we adapted the method for haptic rendering.

The method starts with the quasi-static equilibrium on each node of the FEM mesh:

$$
-f(x)+f_{\text {ext }}+H^{T}(x) \lambda+J^{T}(x) \gamma=0
$$

where $f$ are the internal elastic forces computed by FEM, $f_{e x t}$ are the external forces (i.e. the gravity), $H^{T}$ is the matrix of direction of actuator forces and $\lambda$ is the intensity of the force provided by each actuator. $J^{T}(x) \gamma$ represents the force applied by the user on the device. $J^{T}(x)$ is the direction of the force that we suppose to be known and $\gamma$ is the intensity of this force.

$J^{T}(x) \gamma$ is provided by the virtual/augmented world using contact response process (it corresponds to the value of vector $\mathbf{f}$ in Figure 1) and can be considered as a known external force. We separate it from $f_{\text {ext }}$ to highlight its role in the optimization.

To model the device, the FEM model used is a NeoHookean hyperelastic model computed using Multiplicative Jacobian Energy Decomposition [18]. It relates the internal energy $U$ of the material to the first invariant $I_{1}$ of the right Cauchy-Green deformation tensor and the determinant $\mathcal{J}$ of the deformation gradient through :

$$
U=C\left(I_{1}-3-2 \ln \mathcal{J}\right)+D(\ln \mathcal{J})^{2}
$$

where $C$ and $D$ are material constants related to the shear modulus $\mu$ and bulk modulus $\kappa$ of the material through :

$$
C=\frac{\mu}{2} \text { and } D=\frac{\kappa}{2}
$$

At the beginning of each simulation step, $x$ and $\lambda$ are both unknown and will be solved by optimization. To simplify this optimization, we proceed with a linearization of the internal elastic forces at each step of simulation :

$$
f\left(x_{i}\right)=f\left(x_{i-1}\right)+K\left(x_{i-1}\right) d x
$$

In equation 1 , we replace the value of $f(x)$ by $f\left(x_{i}\right)$. Note that we also use an other simplification of the actuation force expression: we replace $H(x)$ by $H\left(x_{i-1}\right)$. Both simplifications are based on the fact that in quasi-static equilibrium $x_{i-1} \approx x_{i}$ (i.e. there are only small displacements between two steps). We then obtain :

$$
K\left(x_{i-1}\right) d x=-f\left(x_{i-1}\right)+f_{e x t}+H^{T}\left(x_{i-1}\right) \lambda+J^{T}(x) \gamma
$$

By defining $d x=d x^{\text {free }}+d x^{\text {constraint }}$ where $d x^{\text {free }}$ is calculated for $\lambda$ and $\gamma$ equal to zero and $d x^{\text {constraint }}$ is obtained for the following case :

$$
K\left(x_{i-1}\right) d x^{\text {constraint }}=H^{T}\left(x_{i-1}\right) \lambda+J^{T}(x) \gamma
$$

Then, we project the mechanics in the constraint space to describe the motion of the effector position. More precisely, we define $\delta$ which is the gap between the effector position in the simulation and the one measured on the device. With $x^{\text {free }}$ being the position without actuation and though a linearization of $\delta$, we have :

$$
\begin{aligned}
\delta(x) & =\delta\left(x_{\text {free }}+d x^{\text {constraint }}\right) \\
& =\delta^{\text {free }}+J\left(x_{i-1}\right) d x^{\text {constraint }}
\end{aligned}
$$

With $J=\frac{\partial \delta(x)}{\partial x}$ and $\delta^{\text {free }}$ as the value of the gap when $\lambda$ and $\gamma$ vanish. We finally obtain :

$\delta(x)=J\left(x_{i-1}\right) K\left(x_{i-1}\right)^{-1}\left(H^{T}\left(x_{i-1}\right) \lambda+J^{T}(x) \gamma\right)+\delta^{\text {free }}$

We note $W=J\left(x_{i-1}\right) K\left(x_{i-1}\right)^{-1} H^{T}(x)$. The force applied by the user $J\left(x_{i}\right)^{T} \gamma$ will act as a modification of $\delta^{\text {free }}$. We can write:

$$
\delta^{u s e r}=J\left(x_{i-1}\right) K\left(x_{i-1}\right)^{-1} J^{T}(x) \gamma+\delta^{\text {free }}
$$

Which then gives :

$$
\delta=W \lambda+\delta^{u s e r}
$$

The role of the optimization will be to find the values of $\lambda$ (actuator values) that compensate $\delta^{\text {user }}$. By doing so, it will create an interaction force $J^{T}(x) \gamma$ between the user and the device (if the positioning provided by the device is accurate)

We will thus solve the following QP:

$$
\min \left(\frac{1}{2} \delta^{T} \delta\right)=\min \left(\frac{1}{2} \lambda^{T} W^{T} W \lambda+\lambda^{T} W^{T} \delta^{u s e r}\right)
$$

When the QP is solved, we obtain the values $\lambda$ which are then sent to the control of actuators. In the simulation, this value of $\lambda$ allows to compute the position $x_{i}$ that will be used in the following step. Additional inequalities, like $\lambda \geq 0$ can be used when solving the QP in order to account for an accurate modeling of the cables, for instance.

\section{COMMUNiCATION With THE VIRTUAL ENVIRONMENT AND THE DEVICE}

In the virtual environment, a proxy of the user finger is simulated. The finger of the user is tracked on the device, so when it comes into contact with the real device, the proxy comes into contact with a virtual deformable object that is simulated using physics. From the collision response, a value of contact force is computed using the god-object method combined with linear complementarity problem (LCP) technique (see [5] for more details). This force will depend on the intensity of displacement of the touch point inside the object. The law between the contact force intensity and the displacement of the touch point characterize the apparent stiffness of the object we want to emulate. We do not have any assumption on this apparent stiffness law: it can be as simple as a virtual spring stiffness and be equal to a constant 
ratio or more complicated, that is to say nonlinear such as realistic objects behaviors.

Thereafter, the desired contact force, computed in the virtual environment, is sent to the inverse model (value $J^{T}(x) \gamma$ in the section about the inverse model).

The communications between the virtual environment, the inverse simulation and the device can be summarized in the algorithm 1.

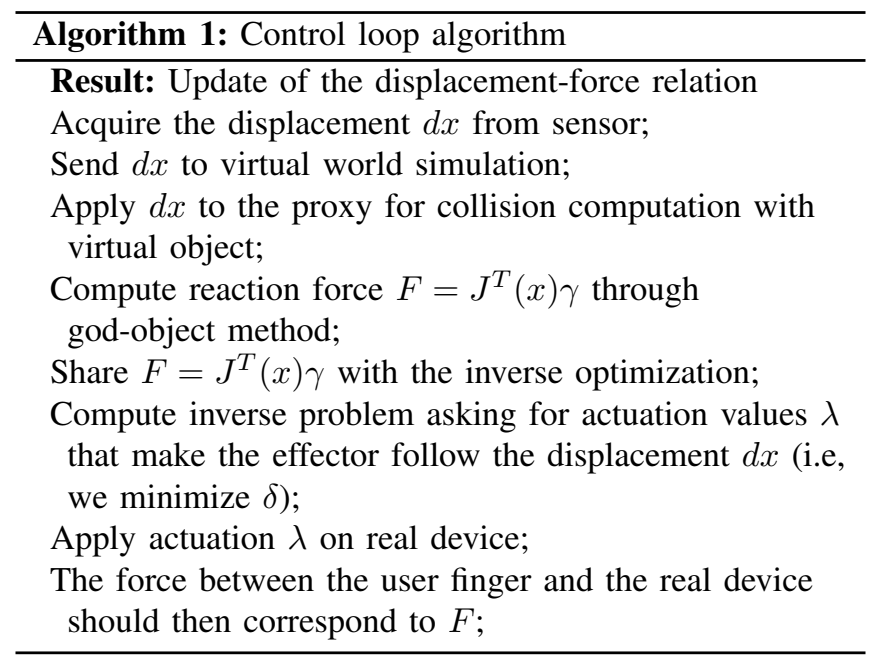

\section{DEVICES}

To demonstrate the genericity of our approach, two devices are tested. We present them in more detail in this section

\section{A. Device based on silicone stretching}

The first device is based on a silicone piece that is connected to motors on both ends and that can pull antagonistically to increase the apparent stiffness of the silicone piece. We use a displacement sensor based on a thin straight rod attached to the finger and sliding without friction in front of an optical mouse sensor with a resolution of 1200dpi and a refresh rate of $125 \mathrm{~Hz}$ to measure the relative positions between the finger of the user and the device. However, the method is not dependent on the type of sensor as long as its refresh rate and precision are sufficient. When the user touches the silicone (Fig 2), the position corresponds to the position of touch in the simulation.

Our prototype is composed of a thin $20 \mathrm{~cm}$ long piece of silicone (Dragon Skin(c)10, Smooth-On, Inc., shear modulus of $45 \mathrm{kPa}$ and bulk modulus of $430 \mathrm{kPa}$ ). The silicone is pulled on each side by a 3D printed clip attached to a cable that is actuated by a Direct Current (DC) motor. An encoder with 512 points per turn provides the effective rotational displacement of each motor. The cables used are made of metallic wire and their elasticity is considered negligible in comparison with the elasticity of the silicone. The rest of the mechanical hardware is composed of aluminum bars and $3 \mathrm{~d}$ printed connective elements. All 3D printed parts of the prototype are made of PLA plastic.
To compute the inverse model, we accurately model the behavior of the device using hyperelastic FEM model of the silicone. The model contains 765 vertices and 400 hexahedra. On a Intel Core i 7 with 8 cores at $3.40 \mathrm{GHz}$, the simulation runs at 34 FPS. We also model the ball bearings that allow to put the motors below. Their behavior is simulated through penalty constraints that bring the silicone back to the surface of the ball bearing when it falsely would penetrate it. This solution offers a more compact design but also maintains the piece of silicone from not falling apart when not actuated. However, this actual design is far from optimal but was chosen in order to focus on the stretching actuation. This example also demonstrates that the inverse model can be computed on a simulation where contacts between parts need to be considered.

An important point for this device is that we impose the same motion and the same actuation force $\lambda_{1}=\lambda_{2}$ for the two actuators in the inverse problem. Indeed, if $\lambda_{1} \neq \lambda_{2}$ then the device could have lateral motions that are difficult to control and prevent.

When the problem is solved, we obtain the actuator force that maintains the position of the device at the input position and we render to the user the force computed in the simulation.

The 2 DC motors of this prototype are controlled by a board that allows to control them in position with proportional regulation.

One of the defaults of the current design of this interface is that we cannot actively control the movement in the downwards direction.

\section{B. Device based on heterogeneous actuation}

The design of the second device is still based on an antagonistic principle. It combines two types of actuators: pressured air and cables piloted by servo motors, similar to the actuation presented in [19]. To create an upwards motion, we use a cavity filled with pressured air (Fig 3 and 4). The pressure inside the cavity is regulated. In the inverse problem, one of the values of $\lambda$ is the pressure inside the cavity. To create a downwards motion, we use 3 cables (tendons) positioned at $120^{\circ}$ and linked to servo-motors placed below the device ${ }^{1}$.

The shape of the device was designed to facilitate extension when the cavity is pressurized. The device is made of the same silicone as the first prototype. The design is made under CAD software and inner and outer molds are therefore designed accordingly. These molds are obtained by 3D printing with PLA plastic. The pneumatic device has to be molded in two parts in order to shape the inner cavity that outer molds alone would fill. The two halves are then bonded using the same silicone to seal the cavity. The device deformations are modeled with 3D FEM, but in that case, we used a linear constitutive law, as the material strain is less important. Indeed, the elongation of this device relies more

\footnotetext{
${ }^{1}$ In figures 3 and 4, the servo-motors and the pneumatic compressor are hidden below the device
} 

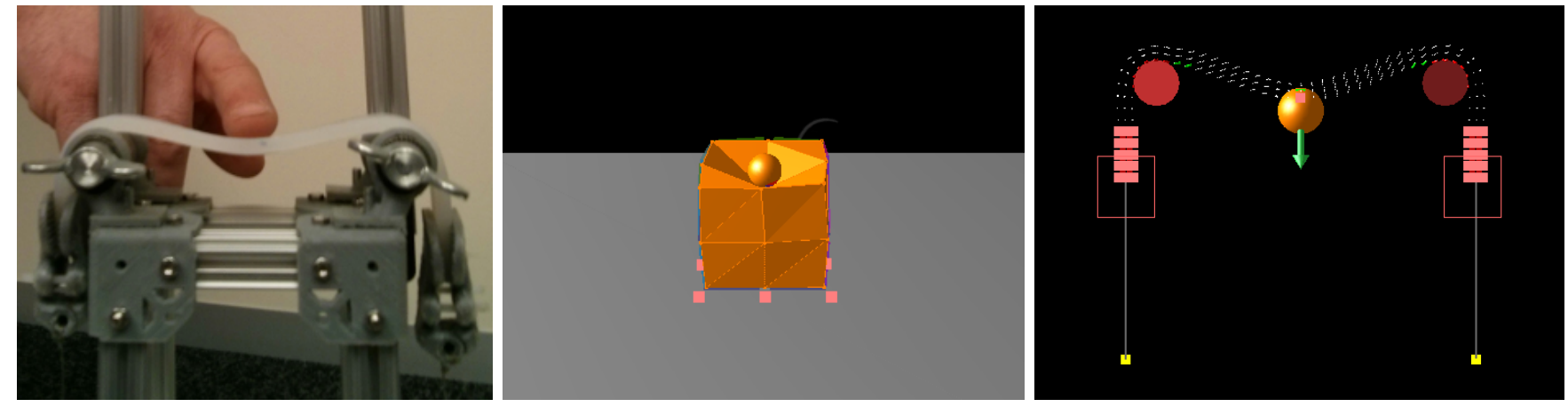

Fig. 2. (left) The first device is conceptually very simple: a thin rectangular piece of silicone is actuated by antagonistic cables placed at its extremities. As part of the device, we measure the displacements of the finger of the user through an optical tracker. (middle) These displacements are used to move a proxy of the finger of the user in the virtual environment, which creates a collision with a soft cube. (right) The contact force is extracted from the simulation and sent to the inverse model method that computes the actuator inputs

on the unfolding of its accordion pattern than on extreme elongation of the silicone. The model contains 858 vertices and 2743 tetrahedra. On the same setup as the first prototype, it runs at 36 FPS.

This design provides some advantages:

1) A larger spectrum of simulated stiffness can be rendered: for instance, there is a factor of 10 in the Young Modulus, between the simulated soft cube in figure 3 and in figure 4.

2) The device can be actively controlled in both directions (upwards and downwards)

3) The orientation of the upper part of the device can also be controlled thanks to the 3 servo-motors. This advantage has not been exploited yet and would require a displacement sensor that can measure in the 3 dimensions.

The method has been successfully applied to both devices. It demonstrates a good genericity of the proposed method.

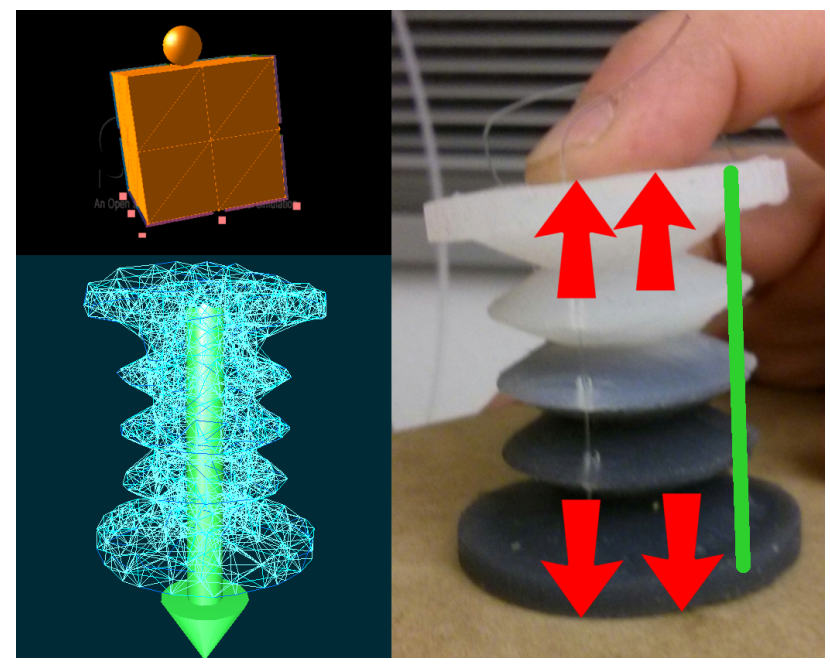

Fig. 3. Second prototype in the case of rendering a stiff object. On top left, the virtual object does not allow easy pressing. The FEA model visible on bottom left provides the force to be rendered and the real device visible on the right is actuated with a large pneumatic pressure. One of the tendons is colored in green for a better understanding of their positioning.

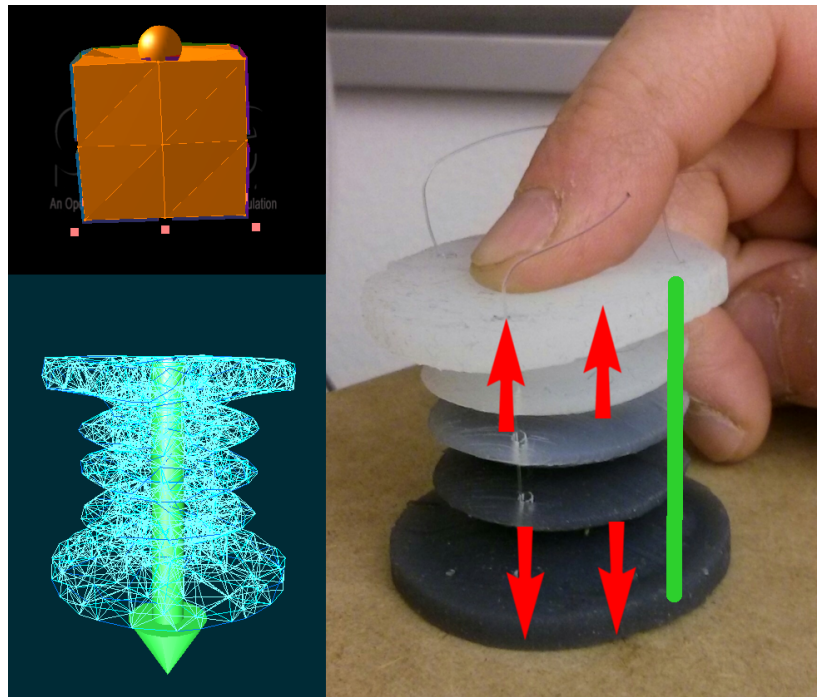

Fig. 4. Second prototype in the case of rendering a soft object. On top left, the virtual object can be pressed easily. The FEA model visible on bottom left provides a force to be rendered that is slightly smaller than in the previous case and the real device visible on the right is actuated with a lesser pneumatic pressure.

\section{DISCUSSION}

This work aims at proposing a new approach for haptic rendering in which the user can touch a soft device on which the stiffness properties are controlled. This approach is very different and complementary from the standard concept of the stylus at the end of a robotic arm. The main contribution of this paper is to show that we can control such hardware by the resolution of a problem built from the inverse of a FEM model. The method is quite generic and has been applied to 2 different devices.

However, we have also observed the following limitations:

- For the control of the silicone stretching device, we could probably use a more simple approach to control the device, with a simple model based on measurements. However, this method would then not be generic and would be more complex to use for the second device, with 4 independent actuators. 
- We use a quasi-static assumption, that restrict the use of the method to slow motions. Indeed, the transient motions are ignored, and, in practice, we have observed that they can perturb the rendering of the device, mostly in the case of the silicone stretching device.

- For proper operation of the method, highly accurate position measurement is needed and at high rates. Indeed, it is important to have the same positioning, with no delay, in the environment and on the device so that contact events appear synchronously. Otherwise the obtained haptic rendering is not transparent.

Moreover, this paper only presents a qualitative validation of the method as the results can only be compared between different actuations that offer a feeling of a softer or stiffer material to the user. In order to present a quantitative validation of the method, it is necessary to use a sensor that can measure both the force rendered and the displacement of the interface. Such a device could be developed from a strain gauge placed on a linear slider with a means to measure the position. However, this mechanical development requires additional investigation to ensure the validity of the measurements that the sensor would provide.

\section{CONCLUSION AND FUTURE WORK}

The work presented in this paper allows the haptic rendering of contact with simulated soft objects. We propose to control a soft haptic device using an inverse FEM model of this device, computed in real-time. We have described the shared information and the links (tracked motion of the finger, contact force, actuator forces) between the three main components: virtual simulated world, inverse simulation and hardware. The main contribution is the antagonistic actuation combined with the inverse simulation algorithm that are used to control the apparent stiffness of the device.

Many improvements can be realized: in a short term, we need to provide experimental data with a pressure measurement device just under the finger in order to characterize the transfer function between computed force to be rendered and real force felt by the user. The analysis of these measurements will also provide elements to define the limitations in performance of the current algorithm, sensors and prototypes. In a middle term, we could propose other types of design and investigate dynamic modeling to control transient motions. In the long term, this work could be used in the conception of a new generation of interactive simulators for training in palpation, medical and surgical procedures.

\section{ACKNOWLEDGMENT}

The authors would like to thank Alexandre Kruszewski, Bruno Carrez and Thor Morales Bieze for their advices on the technical aspects of this project.

\section{REFERENCES}

[1] V. Hayward, O. R. Astley, M. Cruz-Hernandez, D. Grant, and G. Robles-De-La-Torre, "Haptic interfaces and devices," Sensor Review, vol. 24, no. 1, pp. 16-29, 2004.

[2] M. C. Lin and M. Otaduy, Haptic rendering: foundations, algorithms, and applications. CRC Press, 2008.
[3] Y. Payan, Soft tissue biomechanical modeling for computer assisted surgery. Springer, 2012, vol. 11.

[4] C. Basdogan, C.-H. Ho, M. A. Srinivasan, S. D. Small, and S. L. Dawson, "Force interactions in laparoscopic simulations: haptic rendering of soft tissues." Studies in health technology and informatics, vol. 50, pp. 385-391, 1997.

[5] C. Duriez, F. Dubois, A. Kheddar, and C. Andriot, "Realistic haptic rendering of interacting deformable objects in virtual environments," Visualization and Computer Graphics, IEEE Transactions on, vol. 12, no. 1 , pp. $36-47,2006$.

[6] H. Courtecuisse, Y. Adagolodjo, H. Delingette, and C. Duriez, "Haptic rendering of hyperelastic models with friction," in Intelligent Robots and Systems (IROS), 2015 IEEE/RSJ International Conference on. IEEE, 2015, pp. 591-596.

[7] M. Li, T. Ranzani, S. Sareh, L. D. Seneviratne, P. Dasgupta, H. A. Wurdemann, and K. Althoefer, "Multi-fingered haptic palpation utilizing granular jamming stiffness feedback actuators," Smart Materials and Structures, vol. 23, no. 9, p. 095007, 2014.

[8] C. Duriez, "Control of elastic soft robots based on real-time finite element method," in Robotics and Automation (ICRA), 2013 IEEE International Conference on. IEEE, 2013, pp. 3982-3987.

[9] J. Allard, S. Cotin, F. Faure, P.-J. Bensoussan, F. Poyer, C. Duriez, H. Delingette, and L. Grisoni, "Sofa-an open source framework for medical simulation," in MMVR 15-Medicine Meets Virtual Reality, vol. 125. IOP Press, 2007, pp. 13-18.

[10] H. Ishii and B. Ullmer, "Tangible bits: towards seamless interfaces between people, bits and atoms," in Proceedings of the ACM SIGCHI Conference on Human factors in computing systems. ACM, 1997, pp. 234-241.

[11] S. Follmer, D. Leithinger, A. Olwal, A. Hogge, and H. Ishii, "inform: dynamic physical affordances and constraints through shape and object actuation." in UIST, vol. 13, 2013, pp. 417-426.

[12] R. Niiyama, L. Yao, and H. Ishii, "Weight and volume changing device with liquid metal transfer," in Proceedings of the 8th International Conference on Tangible, Embedded and Embodied Interaction. ACM, 2014, pp. 49-52.

[13] L. Yao, R. Niiyama, J. Ou, S. Follmer, C. Della Silva, and H. Ishii, "Pneui: pneumatically actuated soft composite materials for shape changing interfaces," in Proceedings of the 26th annual ACM symposium on User interface software and technology. ACM, 2013, pp. 13-22.

[14] R. Niiyama, X. Sun, L. Yao, H. Ishii, D. Rus, and S. Kim, "Sticky actuator: Free-form planar actuators for animated objects," in Proceedings of the Ninth International Conference on Tangible, Embedded, and Embodied Interaction. ACM, 2015, pp. 77-84.

[15] A. Shiva, A. Stilli, Y. Noh, A. Faragasso, I. D. Falco, G. Gerboni, M. Cianchetti, A. Menciassi, K. Althoefer, and H. A. Wurdemann, "Tendon-based stiffening for a pneumatically actuated soft manipulator," IEEE Robot. Autom. Lett., pp. 1-1, 2016. [Online]. Available: http://dx.doi.org/10.1109/LRA.2016.2523120

[16] M. C.-S. Yuen, R. A. Bilodeau, and R. Kramer, "Active variable stiffness fibers for multifunctional robotic fabrics," IEEE Robot. Autom. Lett., pp. 1-1, 2016. [Online]. Available: http://dx.doi.org/10.1109/LRA.2016.2519609

[17] F. Largilliere, V. Verona, E. Coevoet, M. Sanz-Lopez, J. Dequidt, and C. Duriez, "Real-time control of soft-robots using asynchronous finite element modeling," in Robotics and Automation (ICRA), 2015 IEEE International Conference on. IEEE, 2015, pp. 2550-2555.

[18] S. Marchesseau, T. Heimann, S. Chatelin, R. Willinger, and H. Delingette, "Multiplicative jacobian energy decomposition method for fast porous visco-hyperelastic soft tissue model," in Medical Image Computing and Computer-Assisted Intervention-MICCAI 2010. Springer, 2010, pp. 235-242.

[19] F. Maghooa, A. Stilli, Y. Noh, K. Althoefer, and H. A. Wurdemann, "Tendon and pressure actuation for a bio-inspired manipulator based on an antagonistic principle," in 2015 IEEE International Conference on Robotics and Automation (ICRA). IEEE, 2015, pp. 2556-2561. 